\title{
A PREEMPTION PACKET RESERVATION MULTIPLE ACCESS (P-PRMA) PROTOCOL FOR MULTIMEDIA INTEGRATED ACCESS IN WIRELESS SYSTEM
}

\author{
N. A. El-Fishawy ${ }^{1}$, H.A. k. Mansour ${ }^{2}$, and S. A. Imam ${ }^{3}$ \\ 1 Department of Communications, Faculty of Electronic Engineering, \\ Menoufia University, Egypt, nelfishawy@hotmail.com \\ 2,3 Department of Electronics and Communications, Faculty of \\ Engineering, Benha University, Egypt, hala.mansour@gmail.com, \\ saidimam@hotmail.com
}

(Received September 2, 2006 Accepted October 1, 2006)

\begin{abstract}
In this paper, we explore, via an extensive simulation study the design and performance evaluation of P-PRMA protocol that multiplexes voice traffic at the talkspurt level to efficiently integrate voice, rt-VBR video and data traffics in third generation picocellular wireless networks. We show the effect of preemption over PRMA by comparing two versions of the protocol one with preemption and the other without preemption. We focus on both MPEG-4 and H.263 coded movies with different encoding qualities. The design objectives include maximizing the system capacity (by finding the optimum permission probabilities of sending contending voice, data, and video) and to provide some guaranteed quality of service $(Q o S)$ to each user based on the traffic type. Two particular elements of QoS are considered here, which are the packet dropping probability, and the maximum transmission delay suffered by each packet. Results obtained show that the performance of P-PRMA is superior when compared to the normal PRMA, especially in case of MPEG-4 and HQ H.263 video streams.
\end{abstract}

KEYWORDS: Multiple-access system, Packet reservation multiple access, Multimedia communications, MPEG-4 and H.263 video.

\section{INTRODUCTION}

With the rapid development of high-performance devices, such as portable computers, personal digital assistants, and portable videophones, the demand for providing multimedia services over wireless personal communication networks (WPCNs) has been rapidly increasing [1]. In order to support these services, a channel sharing media access control (MAC) protocol that provides a high throughput and capacity is required. This protocol should also guarantee different quality of service (QoS) parameters for different types of multimedia traffic.

Transmission of real-time variable bit rate (rt-VBR) video is one of the most challenging problems in the future WPCNs [2]. Real-time video service has delivery 
constraints similar to voice. However, while performance analysis for PRMA has been geared toward using voice activity detection to determine the maximum number of users which can be accommodated on the wireless channel, there has been a little work done on optimizing video delivery so that capacity can be improved. Thus a new MAC protocol that is efficiently integrates rt-VBR, voice, and data traffics, is required to be devised.

In this paper, Preemption Packet Reservation Multiple Access Protocol (P-PRMA), a novel MAC protocol is introduced, which is based on the principles of PRMA, which allows contention between terminals and reservations of periodic time slots.

This paper is organized as follows; Section (II) gives a description of the PRMA protocol. Section (III) presents our (P-PRMA) model. Section (IV) describes the system and its parameters that are used in the simulation study. Simulation results are presented and discussed in Section (V). The paper is concluded in Section (VI).

\section{DESCRIPTION OF PRMA}

The idea of PRMA protocol was proposed by Goodman et al, in [3]. PRMA can be viewed as a merger of the slotted Aloha protocol and TDMA [4]. It enables dispersed terminals to transmit packetized information over a shared channel to a BS. The transmission time scale is organized in frames, each containing a fixed number of time slots. The frame rate is identical to the arrival rate of speech packets. The terminals classify each slot as either "reserved" or "available" according to the feedback received from the BS at the end of each slot.

In the next frame, a reserved slot can be used only by the terminal that reserved it. An available slot can be used by any terminal, not holding a reservation, which has information to transmit to the base.

When a terminal begins to generate periodic information, it contends for the next available time slot. Upon detecting the first packet in the information burst, the base station grants the terminal a reservation for exclusive use of the same time slot in the next frame.

At the end of the information burst, the terminal transmits nothing in its reserved slot. This stimulates a NACK feedback message from the base station indicating that the slot is once again available [5].

For data terminals, PRMA can also transmit multiple packet data messages. Data terminal can contend for more than one slot per frame, and not allowed to make reservation [6].

When a collision occurs, terminals will have to retransmit the packets involved in the collision. To transmit a packet, a terminal must verify two conditions. The current time slot must be "available," and, the terminal must have permission to transmit. Permission is granted according to the state of a pseudo random number generator.

A speech terminal attempts to transmit the initial packet of a burst until the BS acknowledges successful reception of the packet, or until the packet is discarded by the terminal because it has been delayed too long. The maximum packet holding time, 
$\boldsymbol{D}_{\max } \mathrm{s}$, is determined by delay constraints on speech communication. $\boldsymbol{D}_{\max }$ is a design parameter of the PRMA system [3]. If a terminal drops the first packet of a burst, it continues to contend for a reservation to send subsequent packets.

Data terminals store packets indefinitely while they contend for reservations. Therefore, when a PRMA system becomes congested, the speech packet dropping rate and the data packet delay both increase.

When traffic builds up, there are significant numbers of packet collisions and terminals encounter delays in gaining access to the channel. Data sources absorb these delays as performance penalties. Conversations, however, require prompt information delivery; and therefore, speech terminals discard delayed packets. In PRMA this packet loss occurs at the beginning of talkspurts, a phenomenon referred to as front end clipping, which impairs the quality of received speech [4]. The amount of front end clipping, as measured by the packet dropping probability, $\boldsymbol{P}_{\text {drop }}$, is an increasing function of the number of speech terminals sharing the PRMA channel. A key measure of PRMA performance is the number of speech terminals that can share a channel within a given maximum value of $\boldsymbol{P}_{\text {drop }}$.

\section{PROPOSED PROTOCOL}

To show the effect of PREEMPTION [7] on PRMA we build two models, the first one is the basic PRMA with modification to support video (which we refer PRMA without Preemption) and the second is PRMA with preemption which we refer PreemptionPRMA (P-PRMA).

\section{A. PRMA Without Preemption:}

In this protocol voice and data terminals behave as in the basic PRMA. For video terminals the BS assigns one slot in each frame for each video user. For simplification we assume one video user. The video terminal transmits the first packet in the video frame $(\mathrm{VF})$ in its reserved slot; also it transmits a request of the number of remaining packets in the current VF along the header. The BS monitors the number of available slots in the channel frame; if the required number is less than the number of available slots the BS will reserve an equal number of slots to the remaining packets in the video buffer. If the required number is greater than the number of available slots; the BS will reserve all available slots to the video terminal and repeat this process again in the next frame for the remaining video packets, and so on until the video delay constraint is reached.

If the video delay constraint is exceeded, the remaining video packets are dropped. If all the packets in the VF have been sent and a new VF is not come yet; the reserved slot for the video terminal is not used.

Since rt-VBR video is variable length coded, the number of video packets varies from frame to frame, and then the dropped packets will be large in larger frames, which will decrease the efficiency especially in case of high quality videos. This led to the next protocol. 


\section{B. Preemption Packet Reservation Multiple Access (P-PRMA):}

To decrease the video dropping probability we use Preemption. When there are remaining video packets and there are no available slots and the video delay constraint will be reached; the BS preempts an equal number of voice users' i.e. takes an equal number of slots reserved for voice users and assigns them to the video user to send its remaining packets before the delay constraint is reached. Then the BS returns back the preempted slots to their voice users in the next frame.

However, each preempted voice user has a packet that is delayed one frame; so if this user is preempted again the packets in its buffer will be delayed by an extra frame and so on. Hence excessive delays will occur, which will cause the voice dropping probability to increase and degradation to the voice service takes place.

To solve this problem we do the following: The BS registers the preempted voice users and after it returns the preempted slots to their voice users it tries to find an extra available slot for each preempted voice user to send its delayed packet. In the same time the preempted user is not allowed to be preempted again until it sends its delayed packet.

Another difference between P-PRMA and PRMA without preemption is that: when all packets in a video frame are sent and the next video frame is not come yet, the BS assigns the reserved video slot to the data user that has the largest number of data packets in its buffer; instead of the reserved slot is to be empty.

Figure 1 shows an example of the P-PRMA In this example; there are 10 slots per frame and frame duration is $20 \mathrm{~ms}$. Let the video frame rate is 25 frames per second i.e. one VF comes every $40 \mathrm{~ms}$. i.e.; a video packet in a VF should not be delayed more than two frames otherwise delayed packets beyond this limit will be dropped.

In Frame X; the feedback information from frame X-1 is recognized as follows: the first slot (0) is reserved for the video terminal, slots $(1,2,5,7,8$ and 9) are reserved for voice terminals, and slots $(3,4$, and 6$)$ are available. If a VF having 8 video packets comes in frame X; the video terminal transmits the first packet in its reserved slot (0), also transmits the number of remaining packets, in its buffer, along the header, i.e. 7 packets. The BS checks if the video delay constraint is reached or not. The BS assigns the 3 available slots to the video user. At the end of frame $X$, the video terminal has sent 4 packets, one on the reserved slot (0) and 3 on the assigned slots $(3,4$, and 6$)$ and hence there will be 4 video packets remaining in the video terminal buffer which delayed $20 \mathrm{msec}$ and the next frame is the last chance to send them.

In frame $X+1$, the feedback information from frame $X$ is recognized as follows; the first slot is reserved for the video terminal, slots $(1,5,7$, and 8) are reserved for voice terminals, and slots $(2,3,4,6$ and 9$)$ are available. Two voice users changed to silent. The video terminal transmits one from the 4 remaining packets and a request for the other 3 in its reserved slot (0). The BS assigns slots (2, 3 and 4$)$ for the remaining 3 packets. Other voice and data terminals can contend to gain access on the remaining available slots. As shown in Fig. 1, a data terminal success to gain access in slot (6) and a voice terminal success to reserve slot (9). However the data terminal is not allowed to reserve slots, then after reception of its packet the BS acknowledges that slot (6) is available in the next frame. 
Frame X

\begin{tabular}{|c|c|c|c|c|c|c|c|c|c|}
\hline $\mathrm{R}_{\mathrm{v}}$ & $\mathrm{R}_{\mathrm{s}}$ & $\mathrm{R}_{\mathrm{s}}$ & $\mathrm{A}$ & $\mathrm{A}$ & $\mathrm{R}_{\mathrm{s}}$ & $\mathrm{A}$ & $\mathrm{R}_{\mathrm{s}}$ & $\mathrm{R}_{\mathrm{s}}$ & $\mathrm{R}_{\mathrm{s}}$ \\
\hline 0 & 1 & 2 & 3 & 4 & 5 & 6 & 7 & 8 & 9 \\
\hline $\mathrm{R}_{\mathrm{v}}$ & $\mathrm{R}_{\mathrm{s}}$ & $\mathrm{R}_{\mathrm{s}}$ & $\mathrm{R}_{\mathrm{v}}$ & $\mathrm{R}_{\mathrm{v}}$ & $\mathrm{R}_{\mathrm{s}}$ & $\mathrm{R}_{\mathrm{v}}$ & $\mathrm{R}_{\mathrm{s}}$ & $\mathrm{R}_{\mathrm{s}}$ & $\mathrm{R}_{\mathrm{s}}$ \\
\hline
\end{tabular}

Frame $\mathrm{X}+1$

\begin{tabular}{|c|c|c|c|c|c|c|c|c|c|}
\hline $\mathrm{R}_{\mathrm{v}}$ & $\mathrm{R}_{\mathrm{s}}$ & $\mathrm{A}$ & $\mathrm{A}$ & $\mathrm{A}$ & $\mathrm{R}_{\mathrm{s}}$ & $\mathrm{A}$ & $\mathrm{R}_{\mathrm{s}}$ & $\mathrm{R}_{\mathrm{s}}$ & $\mathrm{A}$ \\
\hline 0 & 1 & 2 & 3 & 4 & 5 & 6 & 7 & 8 & 9 \\
\hline $\mathrm{R}_{\mathrm{v}}$ & $\mathrm{R}_{\mathrm{s}}$ & $\mathrm{R}_{\mathrm{v}}$ & $\mathrm{R}_{\mathrm{v}}$ & $\mathrm{R}_{\mathrm{v}}$ & $\mathrm{R}_{\mathrm{s}}$ & $\mathrm{D}$ & $\mathrm{R}_{\mathrm{s}}$ & $\mathrm{R}_{\mathrm{s}}$ & $\mathrm{R}_{\mathrm{s}}$ \\
\hline
\end{tabular}

Frame $\mathrm{X}+2$

\begin{tabular}{|c|c|c|c|c|c|c|c|c|c|}
\hline $\mathrm{R}_{\mathrm{v}}$ & $\mathrm{R}_{\mathrm{s}}$ & $\mathrm{A}$ & $\mathrm{A}$ & $\mathrm{A}$ & $\mathrm{R}_{\mathrm{s}}$ & $\mathrm{A}$ & $\mathrm{R}_{\mathrm{s}}$ & $\mathrm{R}_{\mathrm{s}}$ & $\mathrm{R}_{\mathrm{s}}$ \\
\hline 0 & 1 & 2 & 3 & 4 & 5 & 6 & 7 & 8 & 9 \\
\hline $\mathrm{R}_{\mathrm{v}}$ & $\mathrm{R}_{\mathrm{s}}$ & $\mathrm{R}_{\mathrm{v}}$ & $\mathrm{R}_{\mathrm{v}}$ & $\mathrm{R}_{\mathrm{v}}$ & $\mathrm{R}_{\mathrm{s}}$ & $\mathrm{R}_{\mathrm{v}}$ & $\mathrm{R}_{\mathrm{s}}$ & $\mathrm{R}_{\mathrm{s}}$ & $\mathrm{R}_{\mathrm{s}}$ \\
\hline
\end{tabular}

Frame $\mathrm{X}+3$

\begin{tabular}{|c|c|c|c|c|c|c|c|c|c|}
\hline $\mathrm{R}_{\mathrm{v}}$ & $\mathrm{R}_{\mathrm{s}}$ & $\mathrm{A}$ & $\mathrm{A}$ & $\mathrm{A}$ & $\mathrm{R}_{\mathrm{s}}$ & $\mathrm{A}$ & $\mathrm{R}_{\mathrm{s}}$ & $\mathrm{R}_{\mathrm{s}}$ & $\mathrm{R}_{\mathrm{s}}$ \\
\hline 0 & 1 & 2 & 3 & 4 & 5 & 6 & 7 & 8 & 9 \\
\hline $\mathrm{R}_{\mathrm{v}}$ & $\mathrm{R}_{\mathrm{s}}$ & $\mathrm{R}_{\mathrm{v}}$ & $\mathrm{R}_{\mathrm{v}}$ & $\mathrm{R}_{\mathrm{v}}$ & $\mathrm{R}_{\mathrm{v}}$ & $\mathrm{R}_{\mathrm{v}}$ & $\mathrm{R}_{\mathrm{v}}$ & $\mathrm{R}_{\mathrm{v}}$ & $\mathrm{R}_{\mathrm{s}}$ \\
\hline
\end{tabular}

Frame $\mathrm{X}+4$

\begin{tabular}{|c|c|c|c|c|c|c|c|c|c|}
\hline $\mathrm{R}_{\mathrm{v}}$ & $\mathrm{R}_{\mathrm{s}}$ & $\mathrm{A}$ & $\mathrm{A}$ & $\mathrm{A}$ & $\mathrm{R}_{\mathrm{s}}$ & $\mathrm{A}$ & $\mathrm{R}_{\mathrm{s}}$ & $\mathrm{R}_{\mathrm{s}}$ & $\mathrm{R}_{\mathrm{s}}$ \\
\hline 0 & 1 & 2 & 3 & 4 & 5 & 6 & 7 & 8 & 9 \\
\hline $\mathrm{R}_{\mathrm{v}}$ & $\mathrm{R}_{\mathrm{s}}$ & $\mathrm{R}_{\mathrm{s} 5}$ & $\mathrm{R}_{\mathrm{s} 7}$ & $\mathrm{R}_{\mathrm{s} 8}$ & $\mathrm{R}_{\mathrm{s}}$ & $\mathrm{R}_{\mathrm{v}}$ & $\mathrm{R}_{\mathrm{s}}$ & $\mathrm{R}_{\mathrm{s}}$ & $\mathrm{R}_{\mathrm{s}}$ \\
\hline
\end{tabular}

Frame $\mathrm{X}+5$

\begin{tabular}{|c|c|c|c|c|c|c|c|c|c|}
\hline $\mathrm{R}_{\mathrm{v}}$ & $\mathrm{R}_{\mathrm{s}}$ & $\mathrm{A}$ & $\mathrm{A}$ & $\mathrm{A}$ & $\mathrm{R}_{\mathrm{s}}$ & $\mathrm{A}$ & $\mathrm{R}_{\mathrm{s}}$ & $\mathrm{R}_{\mathrm{s}}$ & $\mathrm{R}_{\mathrm{s}}$ \\
\hline 0 & 1 & 2 & 3 & 4 & 5 & 6 & 7 & 8 & 9 \\
\hline $\mathrm{R}_{\mathrm{v}}$ & $\mathrm{R}_{\mathrm{s}}$ & $\mathrm{R}_{\mathrm{v}}$ & $\mathrm{R}_{\mathrm{v}}$ & $\mathrm{R}_{\mathrm{s}}$ & $\mathrm{R}_{\mathrm{s}}$ & $\mathrm{D}$ & $\mathrm{R}_{\mathrm{s}}$ & $\mathrm{R}_{\mathrm{s}}$ & $\mathrm{R}_{\mathrm{s}}$ \\
\hline
\end{tabular}

The upper frame is pre-assigned frame, and the lower is the transmitted one

Fig. 1: Illustration of the P-PRMA protocol.

Another situation; since video is variable length coded, another VF comes which has larger number of packets let's say 13 video packets.

In frame $X+2$, the video terminal transmits the first packet in its buffer in slot (0), and a request of another 12 slots is sent along the header. The BS assigns the 4 available slots $(2,3,4$, and 6$)$ to the video terminal. Again at the end of frame $X+2$, the video terminal has sent 5 packets, while 8 packets are remaining in the video buffer which delayed 20 $\mathrm{ms}$ and the next frame is the last chance to send them.

In frame $X+3$, the video terminal transmits the first packet in the buffer in slot $(0)$, and a request for the remaining 7 video packets is sent along the header. The BS checks for the video packets delay and find that this is the last chance to send these 7 packets. The BS assigns the 4 available slots and preempts 3 voice terminals, i.e. takes slots $(5,7$ and 8) from their voice users to the video user, this frame only. After the video terminal has sent all his packets, the BS returns back the preempted slots $(5,7$ and 8$)$ to their voice terminals to use them for exclusive use again. Also the BS tries to find 3 
extra slots in the next frames to these preempted voice terminals, and till this they cannot be preempted again.

In frame $\mathrm{X}+4$, a new VF arrives, let the VF has 5 packets. The BS gives high priority to the preempted users and assigns slots $(2,3$ and 4$)$ to them to send their delayed packets. The BS assigns the remaining available slot (6) to the video terminal, hence it has sent 2 packets and there are 3 remaining.

In frame $\mathrm{X}+5$, the feedback information from frame $\mathrm{X}+4$ is recognized as follows; the first slot is reserved for the video terminal, slots (1, 5, 7,8 and 9) are reserved for voice terminals, and slots $(2,3,4$, and 6$)$ are available. The video terminal has 3 packets; it will send one of them in slot (0) and a request for the other two is sent along the header. And the process continues in the subsequent frames.

\section{SYSTEM MODEL}

Within the microcell, spatially dispersed source terminals share a radio channel that connects them to a fixed BS. The BS allocates channel resources, delivers feedback information and serves as an interface to the MSC. Since the BS is the sole transmitter on the downlink channel, it is in complete control of the downstream traffic, using TDMA to relay information to the users. Thus, we focus on the uplink (mobiles to BS) channel, where a MAC scheme is required in order to resolve the source terminals contention for channel access. We considered a high capacity wireless channel with channel rate 9.045 Mbps [8], [9].

The uplink channel is divided into frames of equal length. Each frame has duration of 12 ms [2], [8], [9], and accommodates 256 slots. Each slot accommodates exactly one fixed-length packet of ATM size that contains 53 bytes with 5 bytes header and 48 bytes of information.

The channel is assumed to be error free and without capture, in order to find the maximum throughput that our protocol can accomplish under various traffic loads. The models that were used to generate the traffic are based on previous studies involving these three traffic types.

\section{A. Voice Traffic:}

The operation of PRMA is based on the speech activity detector SAD being able to reliably detect idle speech segments. Inactive users' time slots are allocated to other users, who become active [10]. The voice traffic model used here is assumed to be slow SAD, which responds only to the principle talkspurts and gaps, with typical mean values of $1 \mathrm{sec}$ and $1.35 \mathrm{sec}$ respectively [6]. The source rate is taken to be $32 \mathrm{kbps}$.

The speech permission probability, $P_{s}$, is a design variable. If more than one terminal transmits a packet in the same time slot, there will be a collision, and the terminals will transmit again after a random delay governed by the permission mechanism. We assumed here that a voice terminal can hold a packet for only two frames i.e. the maximum voice packet transmission delay is $24 \mathrm{~ms}$. The allowed voice packet dropping probability is taken as 0.01 as in [3].

\section{B. Data Traffic:}

Data terminals are assumed to generate packets at random such the average bit rate is $1200 \mathrm{bps}$, and the maximum average data delay is set to $250 \mathrm{~ms}$ as in [4]. In simulation 
each source is assumed to generate data packets according to a passion process at which the inter-arrival time of the data packets is exponentially distributed [6].

\section{Video Traffic:}

In this paper we use the trace statistics of a publicly available library of frame size of long MPEG-4 and H.263 encoded videos, which have been generated at technical university Berlin [11]-[13].

\section{- The video traces used in simulation:}

\section{a) MPEG-4 video streams:}

We have used two coding versions:

(1) High Quality version, with a mean bit rate $765 \mathrm{Kbps}$. After adding headers to the video packets, the video rate increased to a mean value of $850 \mathrm{Kbps}$

(2) Low quality version, with a mean bit rate $152 \mathrm{Kbps}$. After adding headers to the video packets, the video rate increased to a mean value of $165 \mathrm{kbps}$.

The rate of video frames is 25 frames per second. i.e. new video frames arrive every 40 $\mathrm{ms}$, so we have set the maximum transmission delay for video packets to $40 \mathrm{~ms}$ with packets being dropped when this deadline is reached. The allowed video packet dropping probability is set to 0.0001 [9].

\section{b) H.263 video streams:}

We have used the H.263 video traces of the same movies that we studied with MPEG-4 encoding. We have used three coding qualities of the movie:

(1) High quality version with a mean bit rate $445 \mathrm{Kbps}$. After adding headers to the video packets, the video rate increased to a mean value of $497 \mathrm{Kbps}$.

(2) Medium quality version with a mean bit rate $256 \mathrm{Kbps}$. After adding headers to the video packets, the video rate increased to a mean value of $281 \mathrm{Kbps}$.

(3) Low quality version with a mean bit rate $64 \mathrm{Kbps}$. After adding headers to the video packets, the video rate increased to a mean value of $69 \mathrm{Kbps}$.

The inter-frame period in H.263 encoded videos is not constant as in MPEG-4; it is an integer multiple of $40 \mathrm{~ms}$, so the max transmission delay for the video packets in a VF is equal to the time before the arrival of the next frame. Also the allowed video packet dropping probability is set to 0.0001 .

\section{SIMULATION RESULTS AND DISCUSSION}

The performance of the proposed MAC Protocol is studied through simulation programs, written in $\mathrm{C}++$ and Matlab languages. The system parameters that were used in simulation are summarized in Table I. Each simulation point is the result of an average of 10 independent runs, each with $12,802,560$ slots [i.e. 10.002 min video], with the first 1,282,560 slots neglected as warm up for the system.

First finding the best combination of permission probabilities for voice and data terminals:

\section{A. The Voice Permission Probability:}

Figure 2 shows the relation between the voice dropping probability and the number of simultaneous conversations for a system that supports voice only. We find that the best 
voice permission probability $\left(\mathrm{P}_{\mathrm{v}}\right)$ is in the range of (0.03 to 0.04$)$ where the number of users supported by the system is 554 at $\mathrm{P}_{\mathrm{v}}=0.03,556$ at $\mathrm{P}_{\mathrm{v}}=0.035$ and 560 at $\mathrm{P}_{\mathrm{v}}=$ 0.04. But when $P_{v}=0.04$, from Fig. 2, we find that as the number of users increases beyond 570 users, the voice dropping probability increases dramatically. So the best value of $\mathrm{P}_{\mathrm{v}}$ is in the range 0.03 to 0.035 .

Table I: System parameters.

\begin{tabular}{l|c}
\hline \multicolumn{1}{c||}{ Design parameter } & Value \\
\hline \hline Channel Rate $\left(R_{c}\right)$ & $9.045 \mathrm{Mbps}$ \\
\hline Speech Coding Rate $\left(R_{s}\right)$ & $32 \mathrm{Kbps}$ \\
\hline Data Rate & $1200 \mathrm{bps}$ \\
\hline Frame Duration $(T)$ & $12 \mathrm{~ms}$ \\
\hline Slots per Frame & $256 \mathrm{slots}$ \\
\hline Slot duration & $46.875 \mu \mathrm{s}$ \\
\hline Packet size & 53 bytes \\
\hline Overhead $(H)$ & 5 bytes \\
\hline Mean talkspurt duration & $1.0 \mathrm{~s}$ \\
\hline Mean silence duration & $1.35 \mathrm{~s}$ \\
\hline Voice delay limit $\left(D_{\max }\right)$ & $24 \mathrm{~ms}$ \\
\hline Video delay limit & $40 \mathrm{~ms}$ \\
\hline Maximum data packet delay & $250 \mathrm{~ms}$ \\
\hline Maximum voice dropping probability & 0.01 \\
\hline Maximum video dropping probability & 0.0001 \\
\hline Number of video terminals & 1 \\
\hline Number of Speech terminals & Variable \\
\hline Number of Data terminals & Variable \\
\hline Speech Permission probability & Variable \\
\hline Data permission probability & Variable \\
\hline Run time & $12,802,560 \mathrm{slots}$ \\
\hline \hline
\end{tabular}

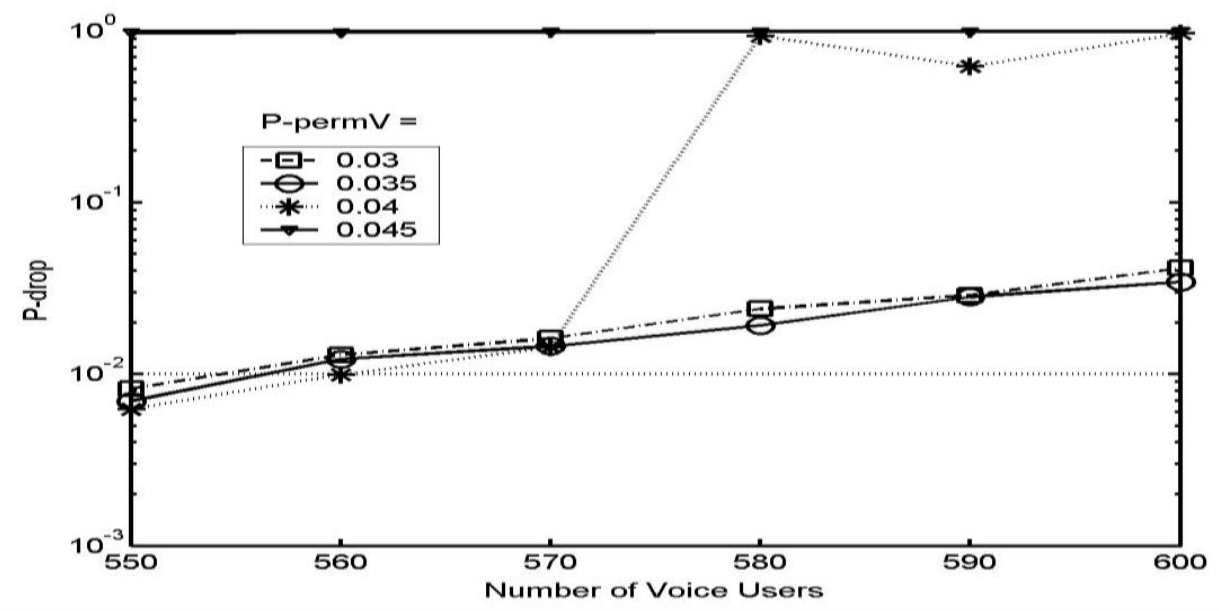

Fig. 2: Voice packet dropping probability vs. the number of simultaneous conversations for voice only PRMA system. 


\section{B. The Data Permission Probability:}

For a system that supports voice and data, we fixed the number of data terminals to 256 terminal; each with a rate of $1200 \mathrm{bps}$.

Several runs have been carried out for various values of voice and data permission probabilities. We find that the best combination of permission probabilities for voice and data that satisfies the QoS requirements are 0.03 and 0.005 respectively. From Fig. 3. we find that the system can support 521 voice users and 256 data users. The channel throughput is 0.891885 .

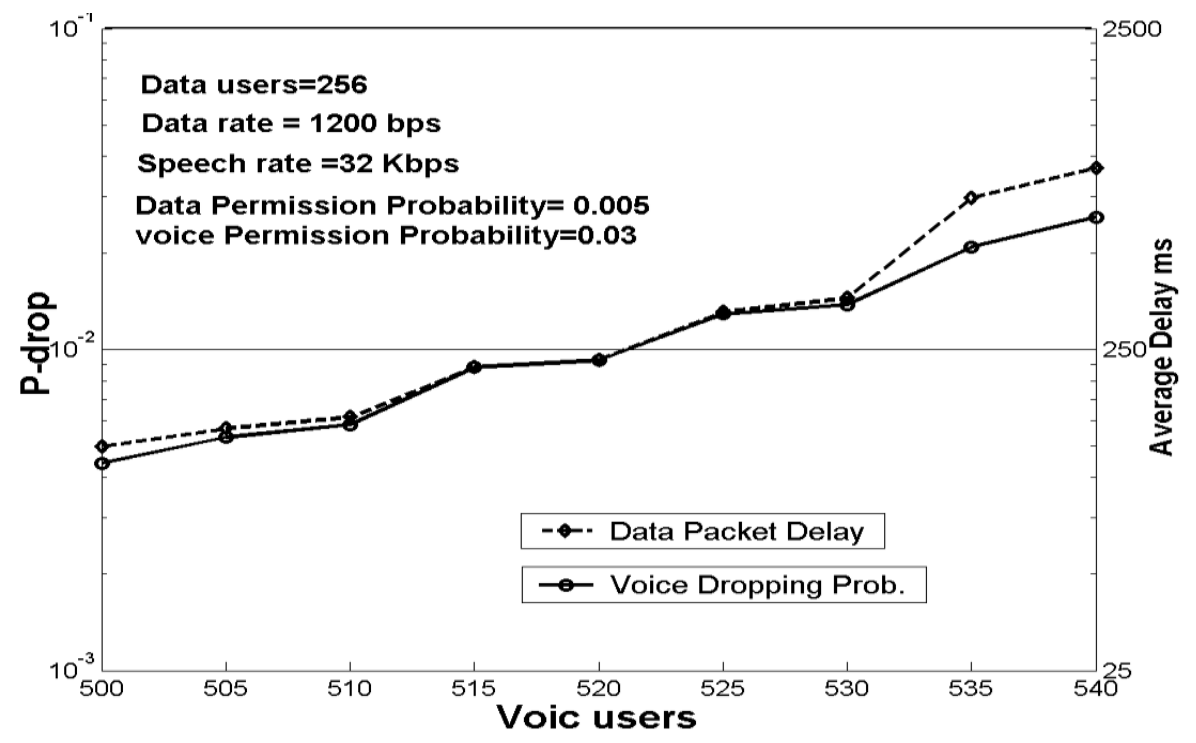

Fig. 3: Voice packet dropping probability and average data packet delay vs. the number of simultaneous conversations for voice/data PRMA system.

\section{The Proposed Protocol Results:}

In this section we try to find the optimum values for the voice and data permission probabilities that provide the maximum system capacity, by determining the number of voice terminals supportable by the system at a fixed number of data terminals and with the different encoding qualities of the video terminal.

The number of data terminals is kept constant at 256 terminals. Several runs have been carried out for various values of voice and data permission probabilities for the two versions of the proposed protocol, the one without preemption and the other with preemption (P-PRMA).

\section{1) Results Obtained For HQ MPEG-4 Video Streams:}

\section{a) PRMA without preemption:}

From the results we obtained that the video dropping probability is the QoS parameter that limits the performance of the system, where it is the first limit that is reached, while the voice dropping probability and the data packet delay are below their limits. 
Figure (4-a) shows the video dropping probability vs. the number of simultaneous conversations at different values of the data permission probability. From the figure, we find that the best result is obtained when the data permission probability is set to 0.005 and the voice permission probability is set to 0.03 , where the system can support 418 voice users, in addition to the 256 data users and the high quality MPEG-4 video user with mean bit rate 850935 bps.

Figure (4-b) shows the voice and video dropping probabilities and the average data packet delay vs. the number of simultaneous conversations at $P_{d}=0.005$. The system throughput is 0.829765 .

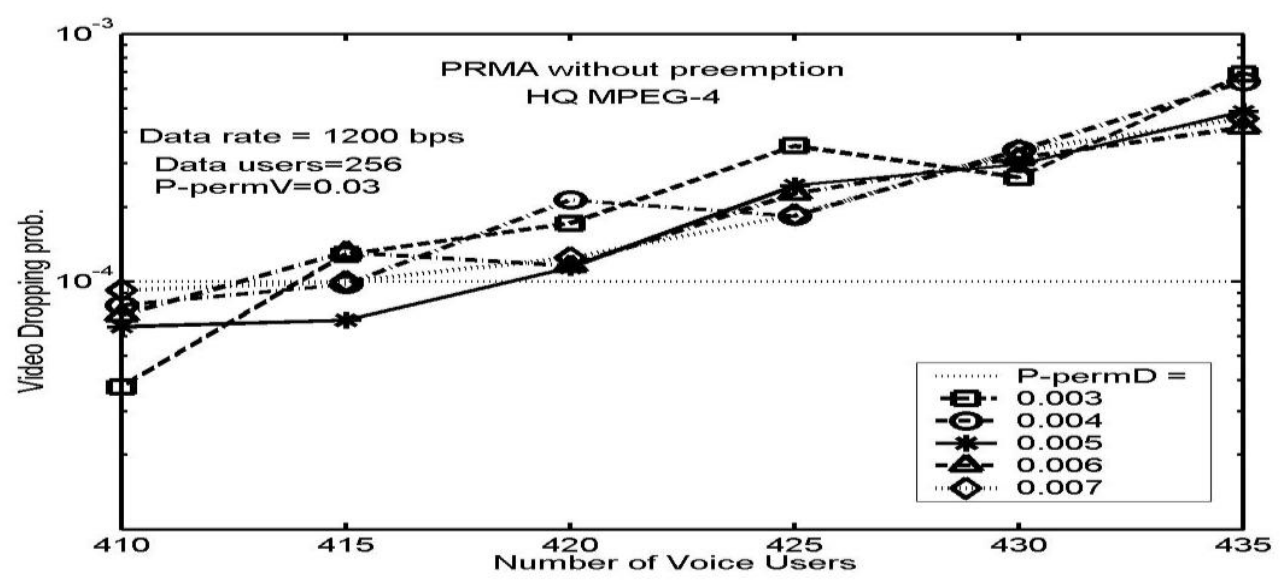

Fig. 4-a: The video dropping probability vs. the number of simultaneous conversations at different values of the data permission probability for PRMA without Preemption (HQ MPEG-4).

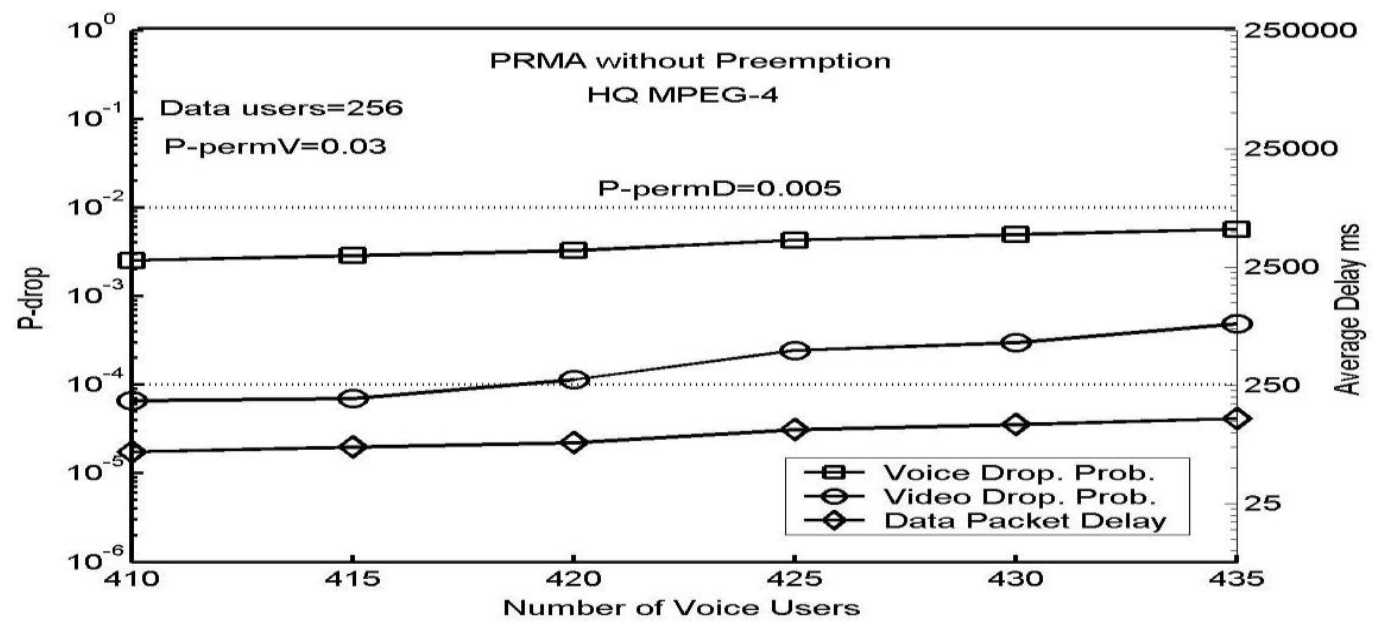

Fig. 4-b: The video dropping probability, voice dropping probability and the average data delay vs. the number of simultaneous conversations at $P_{\text {permD }}=0.005$. 


\section{b) P-PRMA:}

When we applied the preemption algorithm at the same conditions, we find that the system performance has improved than the previous case. First we found that at all points of test that the video dropping probability is zero; i.e. none of the video packets is dropped, as shown in Fig. $\mathbf{5}$.

Second, the best result is obtained when the data permission probability is 0.005 and the voice permission probability is set to 0.03 , where the system can support 446 voice users, in addition to the 256 data users and the high quality video user, i.e. the number of voice users increased from 418 (without Preemption) to 446 (P-PRMA) i.e. by 28 users. The system throughput increased from 0.829765 (NO Preemption) to 0.867739 (P-PRMA).

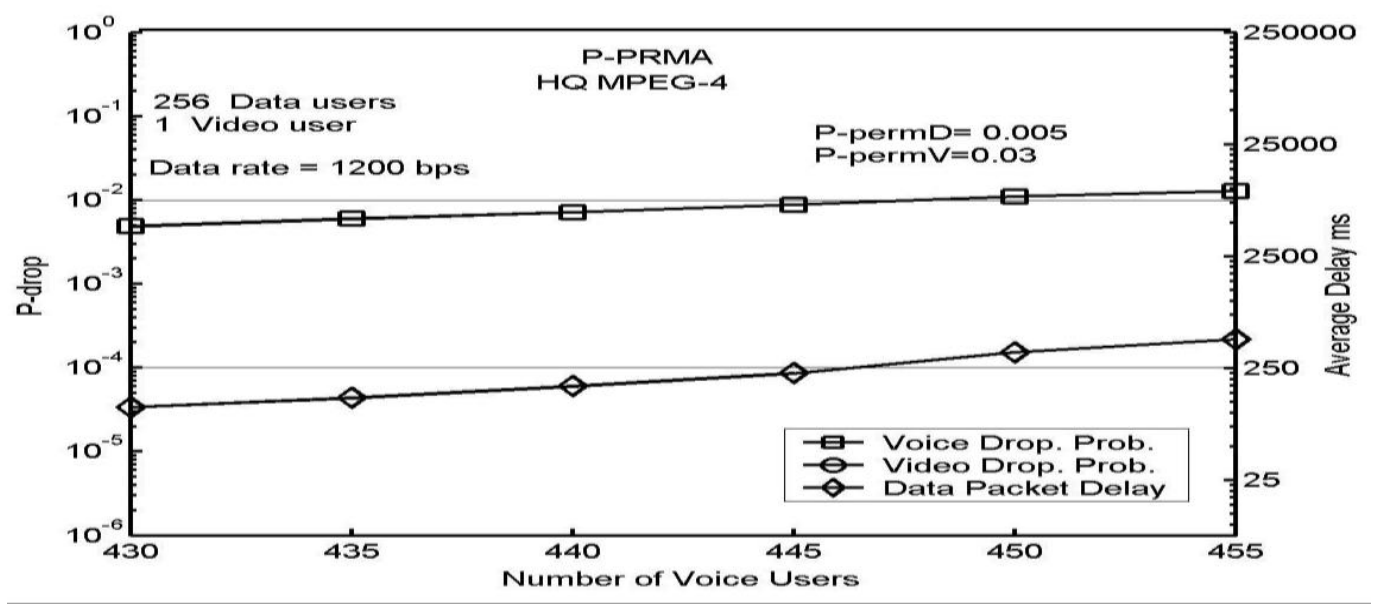

Fig. 5: The video dropping probability, voice dropping probability and the average data delay vs. the number of simultaneous conversations at $P_{\text {permD }}=0.005$ for PRMA with Preemption (HQ MPEG-4); note that the video dropping probability is zero.

\section{2) Results obtained for LQ MPEG-4 video:}

Similar results for HQ MPEG-4 video streams are obtained for LQ video streams, but at higher number of voice terminals.

\section{a) PRMA without preemption:}

The video dropping probability is the QoS parameter that limits the performance of the system. From Fig. 6 we find that the best result is obtained when the data permission probability is set to 0.005 and the voice permission probability is set to 0.03 , where the system can support 477 voice users, in addition to the 256 data users and the LQ MPEG-4 video user with mean bit rate $165 \mathrm{Kbps}$.

\section{b) P-PRMA:}

Here also when we applied the preemption algorithm we found that at all points of test the video dropping probability is zero; i.e. none of the video packets is dropped. The best result is obtained when the data permission probability is set to 0.004 and the 
voice permission probability is set to 0.03 (Fig. 7), where the system can support 510 voice users, in addition to the 256 data users and the LQ video user, i.e. the number of voice users increased from 477 (without Preemption) to 510 (P-PRMA) i.e. by 33 users. The system throughput increased from 0.84552 (NO Preemption) to 0.897867 (P-PRMA).

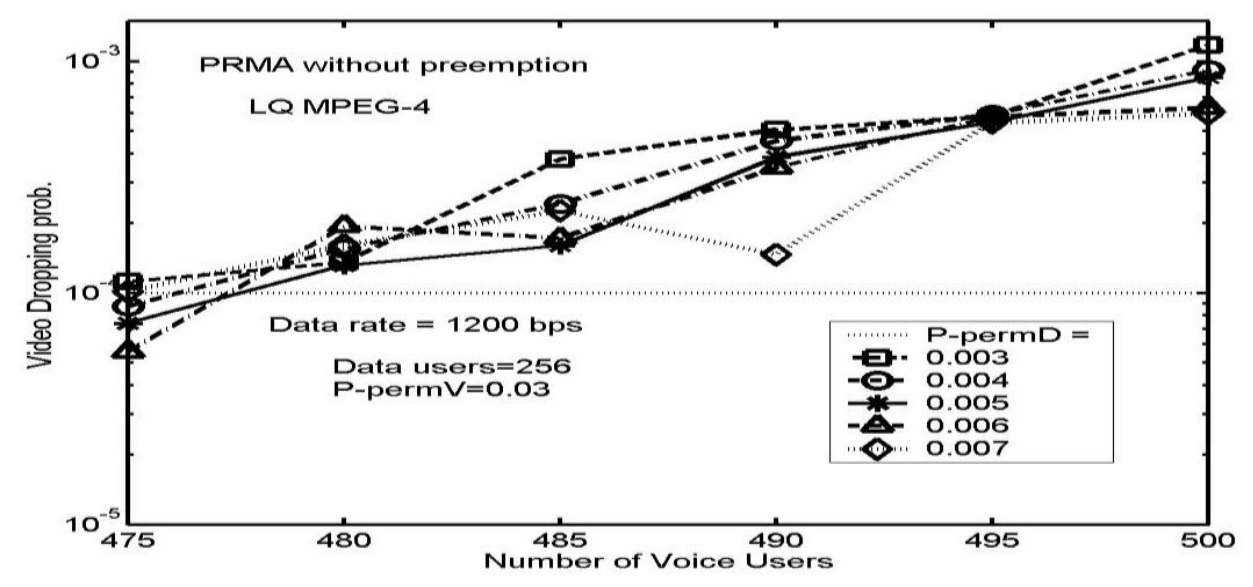

Fig. 6: The video dropping probability vs. the number of simultaneous conversations at different values of the data permission probability for PRMA without Preemption (LQ MPEG-4).

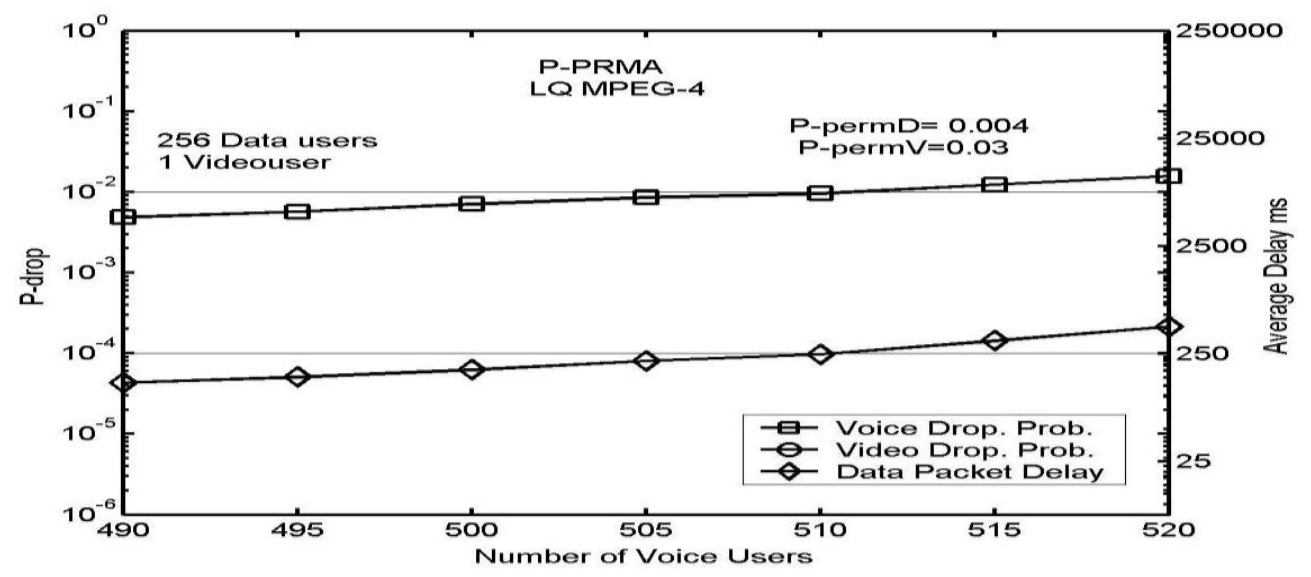

Fig. 7: The video dropping probability, voice dropping probability and the average data delay vs. the number of simultaneous conversations at $P_{\text {permD }}=0.004$ for PRMA with Preemption (LQ MPEG-4); note that the video dropping probability is zero.

\section{3) Results obtained for HQ H.263 video streams:}

The results for HQ H.263 streams are compatible with those obtained for MPEG-4 streams, as follows. 


\section{a) For PRMA without preemption:}

Also we find that the video dropping probability is the QoS parameter that limits the performance of the system. From Fig. 8. we find that the best result is obtained when the data permission probability is set to 0.005 and the voice permission probability is set to 0.03 , where the system can support 426 voice users, in addition to the 256 data users and the high quality H.263 video user with mean bit rate 496868 bps. The system throughput is 0.800635 .

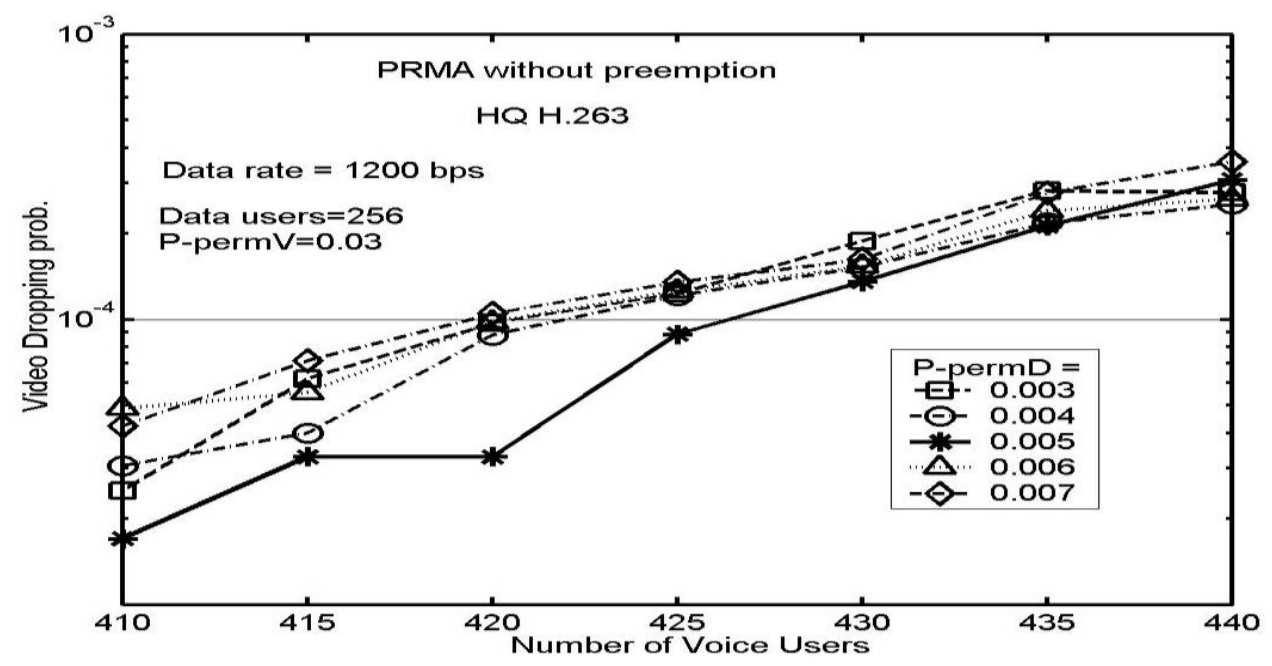

Fig. 8: The video dropping probability vs. the number of simultaneous conversations at different values of the data permission probability for PRMA without Preemption (HQ H.263).

\section{b) P-PRMA:}

When the preemption algorithm is applied we found that at all points of test the video dropping probability is zero. The best result is obtained when the data permission probability is 0.005 and the voice permission probability is set to 0.03 , where the system can support 475 voice users, in addition to the 256 data users and the high quality H.263 video user (Fig. 9), i.e. the number of voice users increased from 426 (without Preemption) to 475 (P-PRMA) i.e. by 49 users. The system throughput increased from 0.800635 (without Preemption) to 0.877573 (P-PRMA).

\section{4) Results obtained for MQ H.263 video streams:}

\section{a) For PRMA without preemption:}

Here there are different results to that obtained for MPEG-4 (both HQ and LQ) and for HQ H.263 streams. Since the video dropping probability is no longer be the QoS parameter that is first reached.

Table II shows the maximum number of voice users at different values of data permission probability and which QoS parameter is reached first. From Table II it is obtained that the best result is when $P_{d}=0.004$, where the system can support 498 voice users in addition to 256 data users and the MQ - H.263 video user with mean rate 
$256 \mathrm{Kbps}$, while the voice permission probability is maintained at 0.03 . Also we find that the voice dropping probability is the QoS parameter that is first reached, while the data packet delay and the video dropping probability are below their limits.

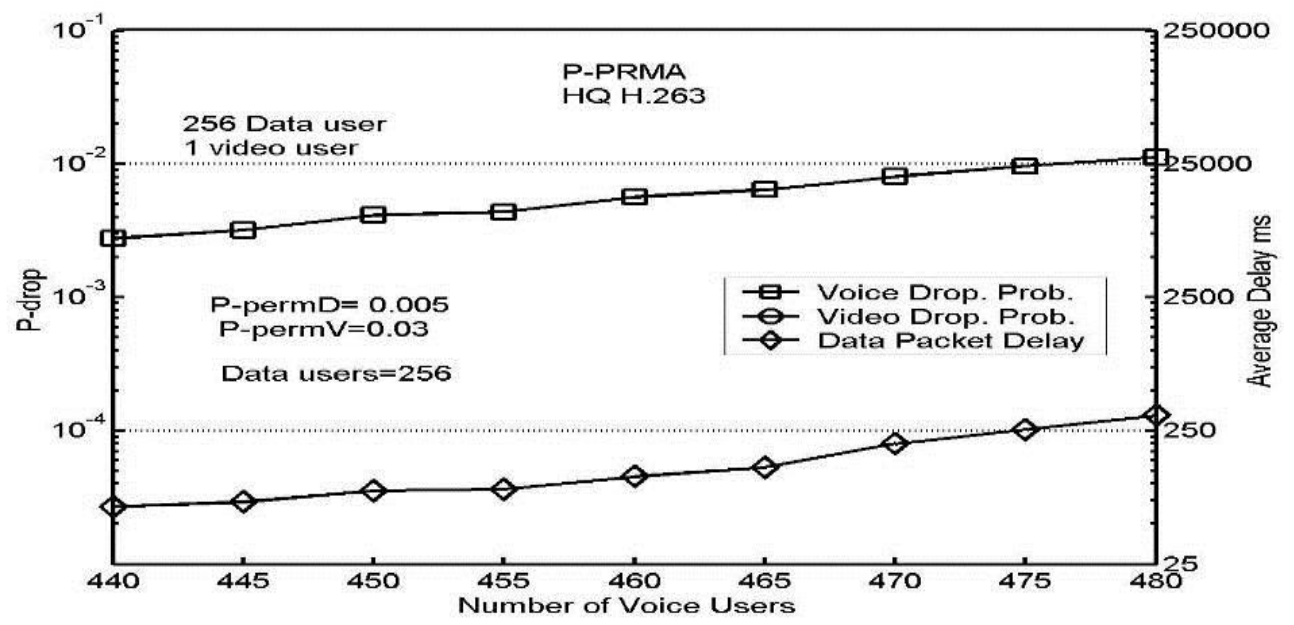

Fig. 9: The video dropping probability, voice dropping probability and the average data delay vs. the number of simultaneous conversations at $P_{\text {permD }}=0.005$ for PRMA with Preemption (HQ H.263); note that the video dropping probability is zero.

Table II: Voice capacity at different values of Data permission probability for PRMA without Preemption for MQ H.263.

\begin{tabular}{l|c|c|c|c|c}
\hline \hline $\mathrm{P}_{\text {permD }}$ & 0.003 & 0.004 & 0.005 & 0.006 & 0.007 \\
\hline \hline Max. voice users & 491 & 498 & 497 & 496 & 495 \\
\hline The limit reached & Data Delay & \multicolumn{5}{|c}{ Voice drop. prob. } \\
\hline \hline
\end{tabular}

\section{b) For P-PRMA:}

When the preemption algorithm is applied a small improvement is obtained in case of the number of voice users while the video dropping probability is improved greatly since at all points of test it has been zero; i.e. none of the video packets has been dropped. Table III summarizes the results in this case.

From table III we find that the best result is obtained when $\mathrm{P}_{\mathrm{d}}=0.004$ as it were in case of no preemption, where the capacity has increased by 2 voice users only.

Table III: Voice capacity at different values of Data permission probability for PRMA with Preemption (P-PRMA) for MQ H.263.

\begin{tabular}{l||c|c|c|c|c}
\hline \hline $\mathrm{P}_{\text {permD }}$ & 0.003 & 0.004 & 0.005 & 0.006 & 0.007 \\
\hline \hline Max. voice users & 490 & 500 & 498 & 497 & 495 \\
\hline The limit reached & Data Delay & \multicolumn{5}{c}{ Voice drop. prob. } \\
\hline \hline
\end{tabular}




\section{5) Results obtained for $L Q H .263$ video streams:}

Similar results are obtained for both protocols as follows:

The video dropping probability at all test points is zero.

The maximum number of voice users obtained at the different values of the data permission probabilities are presented in Table IV.

Table IV: Voice capacity at different values of Data permission probability for LQ H.263.

\begin{tabular}{l|c|c|c|c|c}
\hline \hline $\mathrm{P}_{\text {permD }}$ & 0.003 & 0.004 & 0.005 & 0.006 & 0.007 \\
\hline Max. voice users & 505 & 516 & 515 & 513 & 511 \\
\hline The limit reached & Data Delay & \multicolumn{5}{c}{ Voice drop. prob. } \\
\hline \hline
\end{tabular}

\section{CONCLUSION}

Since MPEG-4 video streams are much burstier and bandwidth consuming than H.263 video streams, we find that for MPEG-4 (in both cases HQ and LQ) and in case of HQ H.263 video streams the video dropping probability is very affecting the performance of the system when the preemption algorithm is not applied. But when the preemption protocol is used, the system performance is greatly improved and the video dropping probability is not affecting the system performance because it is greatly reduced and in all cases it has been zero.

In case of MQ and LQ H.263 we find that the video dropping probability is not the first QoS parameter that affecting the system performance when the preemption algorithm is not applied. This is because that the MQ and LQ H.263 are not burstier as the HQ one and hence less video packets are dropped. So in this case the preemption algorithm is very efficient to reduce the video packet dropping probability to zero, while the system capacity is increased by a very small percentage.

Video packet loss, due to the violation of the maximum video packet transmission delay limit, can result in significant damage of the frame, especially if the dropped packets belong to a VF containing significant information. For example, the loss of an I-frame leads to a decoding error for the whole GoP related to the specific I-frame for both of MPEG-4 and H.263. Hence from the simulation results we can conclude that the proposed P-PRMA protocol is a very efficient MAC protocol suitable for transmitting rt-VBR video streams (MPEG-4 or H.263), voice and data traffics in third generation picocellular wireless networks.

\section{REFERENCES}

[1] J. H. Wen, J. K. Lain, and Y. W. Lai "Performance simulation of wireless multimedia systems using NC-PRMA/DA and SNC-PRMA/DA protocols," IEEE trans. Sys. and human. vol. 32, no. 6, pp. 780-787, 2002.

[2] S. Elnoubi, and A. Alsayh, "A packet reservation multiple access (PRMA)-based algorithm for multimedia wireless system," IEEE Trans. Veh. Technol., vol. 53, pp. 215-222, Jan. 2004. 
[3] D. J. Goodman, R. A. Valenzuela, K. T. Gayliard, and B. Ramamurthi, "Packet reservation multiple access for local wireless communications," IEEE Trans. Commun., vol. 37, pp. 885-890, Aug. 1989.

[4] D. J. Goodman and S. X. Wei, "Efficiency of packet reservation multiple access," IEEE Trans. Veh. Technol., vol. 40, no. 1, part 11, pp. 170-176, Feb. 1991.

[5] S. Nanda, D. J. Goodman and U. Timor, " " Performance of PRMA: a packet voice protocol for cellular systems ," IEEE Transactions on Vehicular Technology, Vol. 40, pp. 584-598, 1991.

[6] H.Y. Chung and D.J. Goodman, "Transmission of speech and data using packet reservation multiple access". IEEE International Conference on Commun., pp. 99-104, 1991

[7] V. Stanisic and M. Devetsikiotis "A Dynamic study of providing quality of service using preemption policies with random election" IEEE International Conference on Commun. Vol. 3, pp. 1543-1546, 2003.

[8] D. A. Dyson and Z. J. Haas, "A dynamic packet reservation multiple access scheme for wireless ATM," ACM/Balzer Mobile Networks and Applications, Vol. 4, pp. 87--99, May 1999.

[9] P. Koutsakis and M. Paterakis, "Call admission control and traffic policing mechanisms for the transmission of videoconference traffic from MPEG-4 and H.263 video coders in wireless ATM networks," IEEE Trans. Veh. Technol., vol. 53, no. 5, pp. 1525-1530, 2004.

[10] L. Hanzo, J. C. S. Cheung, R. Steele, W. T. Webb, "A packet reservation multiple access assisted cordless telecommunication scheme," IEEE Trans. Veh. Technol., vol. 43, no. 2, May 1994, pp. 234 - 244.

[11] http://www-tkn.ee.tu-berlin.de/research/trace/trace.html.

[12] P. Seeling, M. Reisslein, AND B. Kulapala, "Network performance evaluation using frame size and quality traces of single-layer and two-layer and video, a tutorial," IEEE Com. Surveys \& Tutorials, vol. 6, no. 3, pp. 58-78, 2004.

[13] F. H. P. Fitzek and M. Reisslein, "MPEG-4 and H.263 video traces for network performance evaluation," IEEE Network, vol. 15, no. 6, pp. 40-54, 2001.

\section{بروتوكول الولوج المتعدد للحزم المحجوزة باستخدام الأولوية للفيديو لنقل

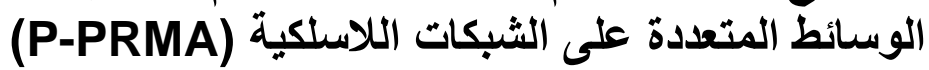

إن التطور الرهيب في الأجهزة الرقيـة عالية الأداء، مثل الحاسبات الآلية المحمولـة وأجهزة

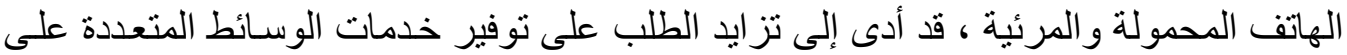

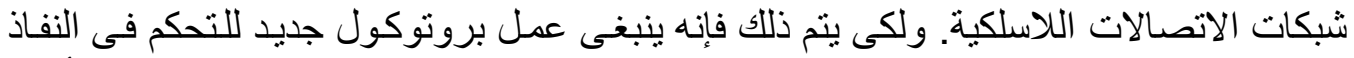

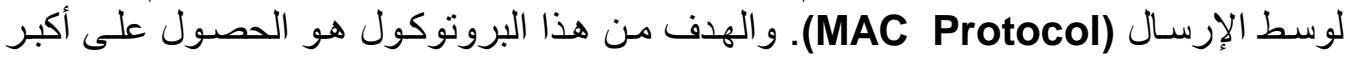

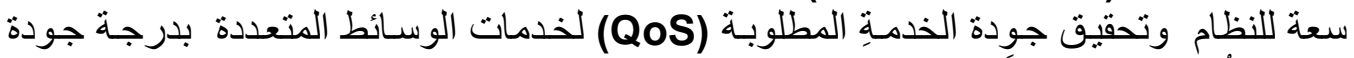

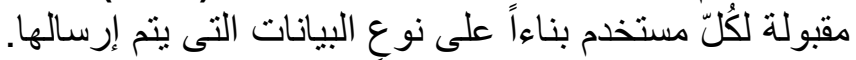




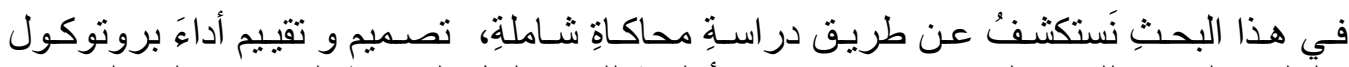

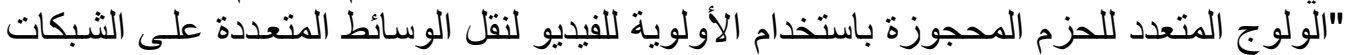

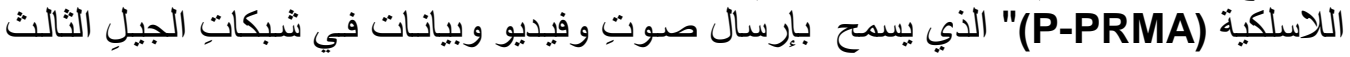

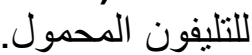
لكي نرى تأثيرَ الأولوية للفيديو (Preemption) فقد قمنا بعمل بروتوكولين، أحدهما يستخدم

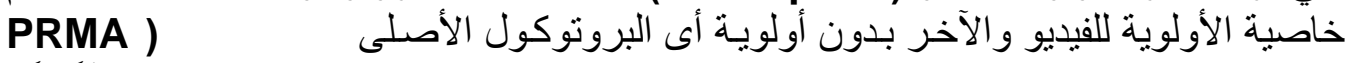
(without Preemption

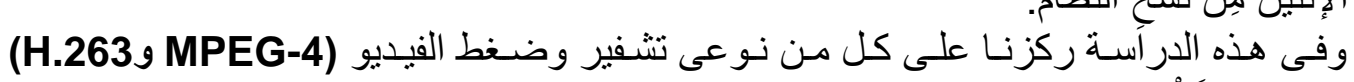

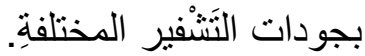

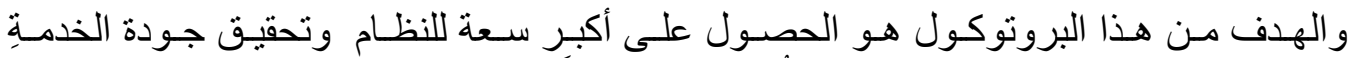

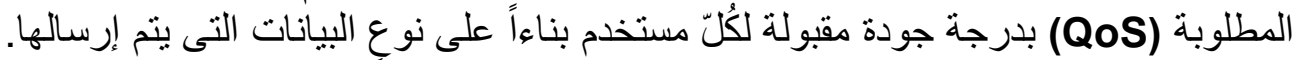

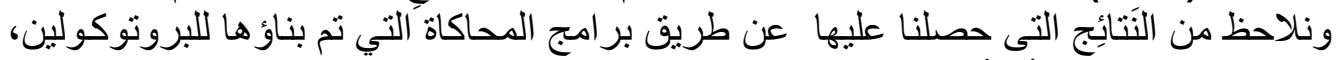

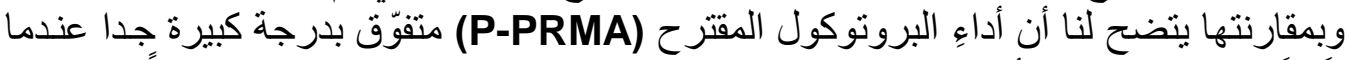

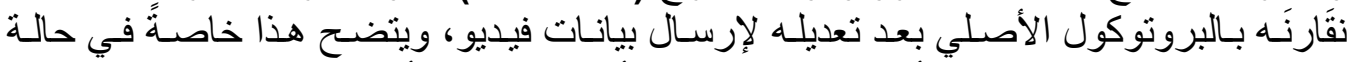
(MPEG-4 video) من (H.263 video). 